Float:

\section{Designing for the Rise in Sea Level}

Camilo Cerro

American University of Sharjah

\section{ABSTRACT: "FLOAT=FLEXIBLE LAND ON AQUATIC TERRITORY" -KOEN OLTHUIS}

According to the United Nations, presently, about 54\% of the world's population lives in urban areas, with the number expected to increase to $66 \%$ by 2050 . Urban areas which are ill prepared to deal with their present population needs will have to develop and manage; housing, healthcare, education, transportation, infrastructure and food production for an additional 2.5 billion people. With three-quarters of the world's megalopolis by the sea and $80 \%$ of people living within 60 miles of the coast, sea level rise will force a new way of thinking about urban development. Managing urban areas has become one of the most important development challenges of the 21st century. In the UAE specifically, there are nearly 1,300 kilometers of coastline. Approximately $85 \%$ of the population and over $90 \%$ of the infrastructure are located within several meters of sea level in low-lying coastal areas. This poses a very specific urban problem of relocation. But not all relocation will need to be done inland. The potential for floating architecture is a very real possibility to help solve some of the problems brought on by the rise in sea level. This is why at the American University of Sharjah, we have been studying this issue and other sustainability related opportunities in a series of courses that started in 2014 with a summer studio course set in Cambodia. Students lived with a floating community in the Tong le Sap lake for a month, studying vernacular floatation systems to inform the development of proposals for floating dwelling studies. This semester (Spring 2018), a fifth year architecture studio set up to transfer specific urban functions to the water within protected areas in the UAE. The aim of the studio was to start looking at possible implementation of floating systems within everyday functions to start a discussion of the potential of this technologies and the feasibility of its use at both an industrial and commercial level. The idea was to develop a series systemic interdependent sustainable designs based on the idea of third nature, hybridizing complex relationships between distinct functions in environments above and below water. This paper will cover the methodology implemented to start tackling these subjects in the studio environment with the aim to create awareness for designers and the general public.

Keywords: Systemic interdependence, third nature, floating dwelling, urban development, vernacular floatation systems.

\section{INTRODUCTION-THE HUNCH:}

In 2013 I had just started working at the American University of Sharjah and was confronted with a program lacking even the basic undertone of sustainability. A subject that is still new for the middle east, it had been adopted more as a trendy fashion than the reality of a design methodology. Presently, this has changed and today the UAE as a whole has taken sustainability as one of their goals, but in 2013 this was not yet the case. As a way of taking on the theme of sustainable design, I implemented it into my studio work at the 300 level. Immediately I realized that the subject was being naively worked with, mainly because the students saw it as an alien element to be dealt 


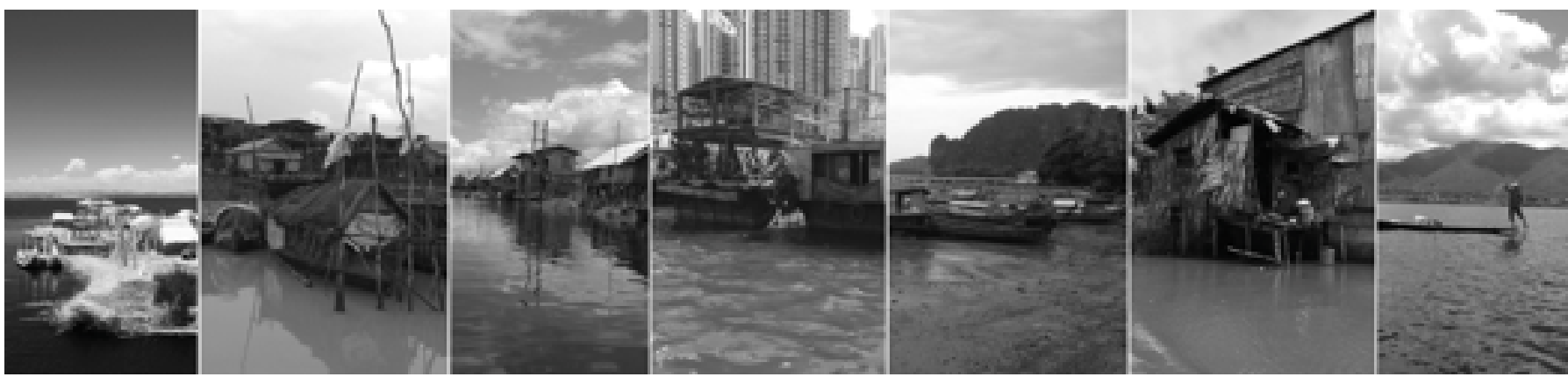

Figure 1. Lake Titicaca-Peru, Lake Tonle Sap-Cambodia, Inle Lake-Myanmar, Aberdeen-Hong Kong, Hoi An-Vietnam, Mekong river-Laos \& Inle lake in Myanmar.

with, much more in terms of a homework assignment than something that could inform their design process. At this point I understood there was a need for a field component where the student could see the social side of sustainable design in person. From this frame of mind is where the hunch originated. How could I implement some of my field work (explained below) into a studio? The hunch ended up being synonymous with personal experience. And my personal experience is in social design, which requires; Site visits, social interaction and material and culture specificity. With this in mind, I coordinated a summer trip for one month to Cambodia where students would be able to design around specific vernacular typologies. During this month, the students lived in a community very different than their own, learning and designing for it. We spent our time around a floating community in the Tonle Sap lake in Cambodia, where the population lives on water. Our goal was to understand their floating dwelling typology, so we could propose our own versions, showcasing what we had learned. Immediately, the issue of bettering the users lives came up and it opened the door to a dialogue about social design, sustainability and quality of life. This teaching methodology kept evolving to produce a studio based approach designed to discuss present issues of global warming and the rise of sea level, not as problems but as design opportunities. This methodology which derives directly from my research and professional practice, has allowed me to produce a well informed subject for academic implementation. The hunch started by bringing the field work into studio to take studio into the field and back again into studio.

\section{INDEPENDENT FIELD WORK:}

The lure of waterfront architecture goes back a long way in human history. But, why has living by water made such an impact on human lives? The reasons vary depending on the population group you are studying, and they are always complex; social, cultural and financial in nature. For the last decade I have been studying vernacular architecture in different communities around the planet with the goal of understanding their relationship to water. This is a list of the places I have documented:

The villages of Joal Fadiouth and Saint Louis in Senegal.

The villages of the Uru people in lake Titicaca and the town of Iquitos in Peru.
The village of Kompong Phluk in lake Tonle sap in Cambodia.

The village of Yawnghwe and Kay Lar Ywa in Inle Lake in Myanmar. Mekong river in Laos.

The village of Hoi An and the Mekong Delta in Vietnam.

The village of Tai $O$ and Aberdeen in Hong Kong.

The research that came out of the field work, looked at present day communities living on water, focusing on urban strategies that have allowed them to function and adapt to the requirements of contemporary life. Everyday tasks are rethought in these communities where boats take the place of cars and elevated walkways that of sidewalks. For generation after generation, these communities have learned to live on water, adapting creature comforts that give them access to: Electricity, healthcare, food, education and all the elements that define a community, while not losing their traditional vernacular typologies. This type of field work, requires time in the communities to gain access and to properly document a way of life. It is as much an anthropological endeavor as it is architectural in nature.

\section{STUDIO DESIGN METHODOLOGY: THIRD NATURE}

The idea of third nature was presented by Flemming Rafn Thomsen and Ole Schrøder of the architecture and landscape firm Tredje Natur from Copenhagen Denmark at an Utzon lecture series in 2014 [1]. For them third nature 'is a holistic perspective, that is based on the idea that the problems of the world cannot be solved by only thinking about the parts of the system and that a framework for a high quality of life is not achieved by solely looking at sustainability, aesthetics, solutions, cities, or nature separately. Nature value is built on the idea that everything in the world is interconnected and we must see the big picture for change to be possible [2].' The floating design proposals which function as the basis for this paper grow from the idea of third nature but to understand this fundamental term we will need to define what first and second nature mean. The idea of first nature refers to the natural world. Sometimes used in a very romanticized way, it proposes the idea of an environment that is interdependent and to some degree in balance. Second nature is defined as the type of growth that takes place when the urban fabric is allowed to expand out of control, in an invasive unplanned way (like how it does 
in informal settlements). When this happens, it always does so at the cost of what we defined as First Nature. And the results are always detrimental to the quality of life of its inhabitants. Third nature then is a symbiotic hybrid that creates a system of interconnected elements designed to create a man-made balance. It is the capacity that design has, to help architecture develop an interdependent system that is able to produce a new type of environment, mixing urban and natural components in a symbiotic way to educate, feed, empower and better the quality of life of the population. This hybrid needs to work at a micro and macro scale. In the specific case of the design projects we are proposing, it needs to function at the residential and the urban levels. And it needs to do so in a manner that benefits the existing environment by solving specific social and ecological issues as a result of its existence.

\section{THE PROGRAM:}

For this type of studio the student is given a level of freedom in terms of the development of their program. They get to propose the subprograms around a given main program. So there is an overall programmatic element that functions interdependently with satellite programs all of them related this main program. Guiding parameters are set for the main program in terms of specific technical themes that need to be addressed on the projects, this include; Power generation and storage, water production, recycling, storage and filtering, local materials and construction capacities and in some cases food production. These themes can be approached in an active or passive manner depending on the students research and the ideas they are interested in developing. The main program is always related to a local need and it is based on extensive site and community analysis. From here the student will develop a proposal that serves as a catalyst for micro programs to function as a systemic interdependent entity. This approach will produce first a macro urban proposal divided into micro components. Depending on the size of the project, the student will decide on how far to develop any of the micro components or whether to instead develop one more than the others. Throughout the semester, the studio offers a series of lectures on sustainable technologies and social design to start a conversation about possible micro-projects and their feasibility, these conversations are always done as a group. This methodology gets reassessed and transformed every term. Following you will see the first and last of this studio as a point of comparison, each explaining the elements that worked and those that needed to be rethought.

\section{THE BEGINNING: CAMBODIA}

In the summer of 2014, I took 16 students to live and work near the Tonle Sap lake in Cambodia. There, we needed a base of operations that would combined proximity to the elements we wanted to research with a safe environment for the students to do so. Because of this, we set shop in the city of Siem Reap, a touristic village near Angkor Wat that had a level of creature comforts that combined recognizable global elements designed for tourists (Markets, restaurants, etc.) with the level of exoticism that comes from visiting a culture very different than their own. For most of our students, Cambodia was not a place they would visit in their everyday life, and probably one they would never visit again. This presented the first problem we had to confront, and had not anticipated. Our students were not very independent and did not know how to function on their own. We saw this as an unrelated opportunity to the program, which was addressed by having social functioning classes that covered how to deal with laundry, how to not pay more than the true value of things, how to treat the local population and staff with respect and how to move around, which was solved by getting everyone a bicycle. Once the crew was mobile, we had no problem visiting communities to do field work and start to understand our site. We visited vernacular architecture and documented how people lived on water. This information, served as the basis for the our studio project themed around floating residential living. Programmatically the students were confronted with a different type of dwelling, one with very different uses than those they were accustomed to, forcing their design to rely on their understanding of our research, which lead to trusting our field work. The students worked individually, producing projects based on local materials and construction methods that utilized technologies (hydroponics, solar energy, toilet composting, aquaponics, etc.) already present in the communities which we visited and saw functioning. The studio was successful in terms of displacing our students from their preconceptions by forcing them to adapt to a new set of rules defined by a culture other than their own. We understood the benefits of this but also saw that we were not going to be able to count on that when reproducing the methodology in a non-displaced studio back in the UAE. It also showed us that the students were eager to learn about sustainability once they saw its potential of bettering the quality of life of users and their communities. The hunch to take students on a field trip-based course was a good one, and even though our work was not as deep and thorough as what I would have done in a non-academic setting, the educational potential for the student was evident. Their work showed an understanding of what they had seen and learned and the experience is one they would not forget. Given that, a month in a single location is still not enough time to do proper field work, but it is good enough to fit the academic calendar.

\section{OUR PRESENT WORK: UAE}

The latest version of the sustainability studio using the methodology presented on this paper took place on the Spring of 2019 (500 level studio). This time, the program would take place in the UAE and the students were asked to choose their sites after proposing the main program for their projects. The sites had to be by the ocean but in protected areas to avoid 
waves. The students had to make their choices with an understanding of topography and proximity to the areas that would benefit from their interventions. Every project ended up having a different site. And to develop better work from the participants, the projects have moved from being individual designs as on the first iterations of the methodology to group projects presently. This has allowed for (as you can see on figures $2 \& 3$ ) much more developed work and more mature dialogue between the group participants. Each group was also asked to explore a number of sustainable technologies that would apply to their programs. The students, visited sites and did field work and research to develop distinct programs related to the everyday life of the Emirates in the fields of hospitality, scientific research and food production. All of which were floating independently or floating but attached to land. The groups had to make models and test them for buoyancy in a water tank, once a floating system proved to function the way the students proposed, it was implemented in the project. Most projects ended up using more than one system, depending on the weight of the program above it. Figures $2 \& 3$ show the final review panels of some of the work produced during the term..

The project shown on figure 2 is located within one of the remaining fishing ports on the UAE. As fish is harder to catch in the ocean, forcing fisherman to travel farther away, this project is proposing a fish farm, packaging and distribution hub to preserve the livelihood of the community by providing jobs that are presently being lost to a dwindling fishing economy and adding a source of food to the country as a whole (the UAE imports $75 \%$ of their food). The facility is attached to the port but designed to float freely in the future once the rise of sea level takes over the coastline. The building also houses a community center and some residences for the staff of the farm. Most of the transport and packaging work is automated. The facility also produces water through atmospheric water generators and energy from solar panels and underwater turbines. The second projects shown on figure 3 is a SPA and hotel facility designed with drone rooms that move automatically during the day to take advantage of the protected area they inhabit. The whole facility is self-sufficient, producing its own energy, water and some of its food. It is located at a midpoint between the cities of Abu Dhabi and Dubai with water taxis in both as means of transport into the facility. Users can also come on their own yachts and use the facility as a marina. The central floating buildings house; restaurants, the SPA, water related activities and the management of the facility while the peripheral ring serves as a port and residential area.

As the students adapted to the parameters of building on water, new representation techniques needed to be implemented to fully explain their projects. One of them, the underwater RCP, which became very useful to describe a new façade that floating architecture needs to deal with, created by the underside of the facility which in a lot of the projects became as important as the plan itself. Modelmaking needed to produce elements that function instead of representational tools only, they needed to float. And because we were working at the micro and macro, the students needed to detail aspects of their project in terms of structure, systems and materiality. What materials can you use next to water? became one the most important questions they had to research, over and over again. All the projects had to also deal with a type of architecture composed of multiple systems, designed for the large proposals to be able to move with the oscillations of the water. I don't believe that the work was better than the one done in other studios, but by 5 th year, students are bored of following the same design and presentation parameters. This project, forced them to think differently bringing back a level of energy normally stagnant at the 501 level.

The studio's submittals were divided on a series of assignments that started with a fieldwork component to choose a site. For this the students studies topographical maps of different parts of the country as they prepared program proposals. Once the sites were chosen, the students documented each of the sites and spent time in them, getting acquainted with them. The sites were visited from land and water. The studio had a first review to talk about programmatic proposals and floating systems. Through the semester, floating models were tested in a water tank. The second review was a gallery style review, allowing the students to have multiple points of view in independent conversations between one reviewer and a student group (two students). This approach gave the students a very different discussion from reviewer to reviewer, very different than the wolf pack review process they normally experience in a traditional review. And finally, the final review was a standard review. Through the semester, the students remained in contact with their sites. We also visited existing floating architecture in Dubai and had multiple lectures on sustainability and vernacular and contemporary floating precedent.

\section{SUSTAINABLE DEVELOPMENT GOALS:}

According to the United Nations, the Sustainable Development Goals are the blueprint to achieve a better and more sustainable future for all. They address the global challenges we face, including those related to poverty, inequality, climate, environmental degradation, prosperity, and peace and justice [3]. Because of this, the studio course implements as many of the goals as they fit in the work produced by students, trying to develop work that produces a systemic interdependence that combines design with social, economic and cultural factors with goal of developing a way of approaching design from a sustainable perspective.

\section{CONCLUSION:}

Going back to the idea of the hunch, I believe there is an inherent second layer to the hunch being just personal experience, and that has to do with the amount of work that comes after having an idea with potential. Because a good idea does not guaranty implementation, for that to happen the methodology needs to be applied in enough studios to allow it to evolve. And for that to happen, the program needs to be compelling enough to engage the students, and it needs to exist within a theme big enough that it can be reapplied over and over again without falling in repetition. In my experience, engaging with field research in studio works best if the program is composed of 

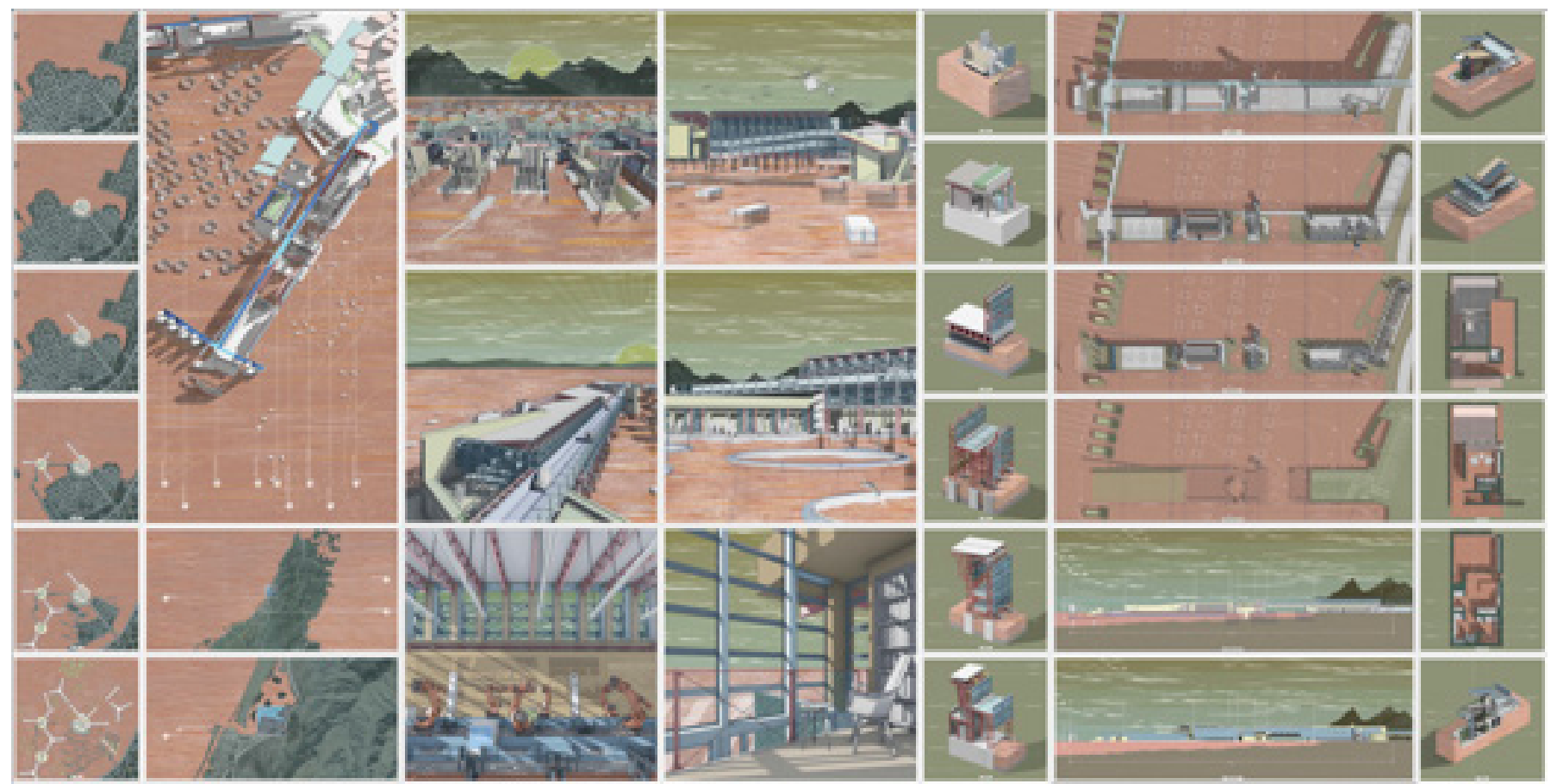

Figure 2: Fish farm and packaging facility with a community center and residential units.

Final review boards by Saad Mohamed Boujan \& Mark Hany Shehata (5th year students).
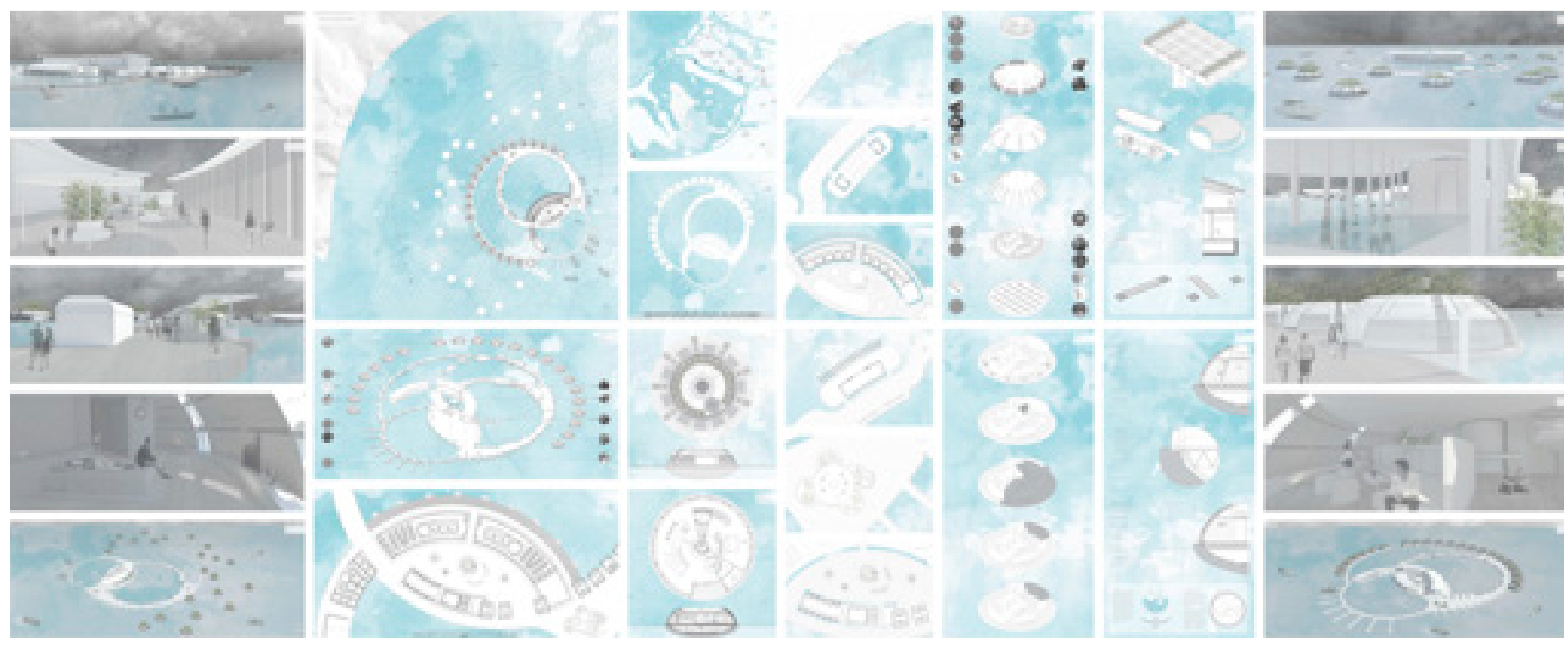

Figure 3: SPA retreat complex and hotel. Final review boards by Sarah Mariam Beebeejaun \& Noora Khalid Alkhazraji (5th year students).

a combination of constrained elements and at the same time, it gives the students enough freedom to explore within those parameters, design ideas that interest them. In the case of this experiment in floating architecture, the larger umbrella is composed of sustainable ideals and technologies, by offering this conceptual undertone, the studio is freed to give the student control over formal and programmatic experimentation which guaranties a plethora of dissimilar work. The hunch then has the potential to evolve to a structured methodology, and if it does this, it will stop being a hunch. Because the place for a hunch is at the beginning of the process, the rest needs to be much more than that.

Pragmatically, this process is serving to add a sustainable 
component to the College of Architecture, Art and Design's ethos. One that is functioning side by side with a push by the government of the UAE to address human made global warming. In the context of the UAE, this type of studio work is starting a dialogue in a country where there are nearly 1,300 kilometers of coastline. And where approximately $85 \%$ of the population and over $90 \%$ of the infrastructure are located within several meters of sea level in low-lying coastal areas, creating a very specific urban problem of relocation and adaptation. Because of this, it becomes very important to start addressing these subjects at the university level as an inherent aspect of the architectural education.

\section{WHAT IS NEXT?}

This methodology has evolved into two research branches; One on water which is the basis for this paper, the other is an exploration of the same sustainable systemic interdependence on land. This second branch will be explored on a vertical studio ( $400 \& 500$ levels) in the Fall of 2019. In this case, the overall grounding component (macro) will be an urban farm which will be interdependently linked to a series of micro-programs to be proposed and developed by the students. The project is designed to help alleviate the food requirements of a community, and at the same time, provide design solutions to other social problems, serving as a catalyst for change.

\section{ACKNOWLEDGMENTS:}

I would like to thank the American University of Sharjah and specially the administration of the College of Architecture, Art and Design for their constant support of my research and academic activities.

\section{REFERENCES:}

1. Flemming Rafn Thomsen, Third Nature. Towards empathetic, transparent and engaging cities, UTZON (x) Lecture series, fall 2014, 13:00, October 8th, Create campus.

2. Agnete Schimmell Raakjær, Ole Schrøder og Flemming Rafn Thomsen, 2017, Nature Value, tredje natur, http://tredjenatur.dk/ en/nature-value/. Accessed: January 13th 2017.

3. United Nations, The Millennium Development Goals Report 2012(UN, 2012). 